\title{
Radical antegrade modular pancreatosplenectomy versus standard distal pancreatosplenectomy for pancreatic cancer, a dual-institutional analysis
}

\author{
Jonathan G. Sham ${ }^{1}$, Shiwei Guo ${ }^{2}$, Ding Ding ${ }^{3}$, Zhuo Shao ${ }^{2}$, Michael Wright ${ }^{3}$, Wei Jing ${ }^{2}$, Ling-Di Yin ${ }^{3}$, \\ Yijie Zhang ${ }^{2}$, Michele M. Gage ${ }^{3}$, Yingqi Zhou ${ }^{2}$, Ammar Javed ${ }^{3}$, Richard A. Burkhart ${ }^{3}$, Xuyu Zhou ${ }^{2}$, \\ Matthew J. Weiss ${ }^{4}$, Tianlin $\mathrm{He}^{2}$, Gang $\mathrm{Li}^{2}$, John L. Cameron ${ }^{3}$, Xiangui Hu ${ }^{2}$, Christopher L. Wolfgang ${ }^{3}$, \\ Gang Jin' ${ }^{2}$ Jin $\mathrm{He}^{3}$
}

${ }^{1}$ Department of Surgery, University of Washington, Seattle, Washington, USA; ${ }^{2}$ Department of Surgery, Changhai Hospital, Shanghai, China; ${ }^{3}$ Department of Surgery, Johns Hopkins Hospital, Baltimore, Maryland, USA; ${ }^{4}$ Northwell Health Cancer Institute, Lake Success, New York, USA

Contributions: (I) Conception and design: JG Sham, S Guo, D Ding, G Jin, J He; (II) Administrative support: None; (III) Provision of study materials or patients: JG Sham, D Ding, Z Shao, M Wright, W Jing, LD Yin, Y Zhang, MM Gage, Y Zhou, A Javed, RA Burkhart, X Zhou, MJ Weiss, T He, G Li, JL Cameron, X Hu, CL Wolfgang, G Jin, J He; (IV) Collection and assembly of data: JG Sham, D Ding, G Jin, J He; (V) Data analysis and interpretation: JG Sham, S Guo, D Ding, M Wright, MM Gage, G Jin, J He; (VI) Manuscript writing: All authors; (VII) Final approval of manuscript: All authors.

Correspondence to: Jin He, MD, PhD, FACS. Department of Surgery, The Johns Hopkins University School of Medicine, 600 N. Wolfe Street, Halsted 614, Baltimore, MD 21287, USA. Email: jhe11@jhmi.edu; Gang Jin, MD, PhD. Department of Surgery, Changhai Hospital, No. 168 changhai road, Yangpu District, Shanghai, China. Email: jingang@sohu.com.

Background: Radical antegrade modular pancreatosplenectomy (RAMPS) has been adopted by some surgeons in the treatment of left-sided pancreatic cancer (PDAC). Low disease incidence and heterogenous disease biology make robust prospective comparison of RAMPS and standard distal pancreatosplenectomy (DPS) difficult.

Methods: Consecutive cases of chemo-naïve patients undergoing open RAMPS and DPS for PDAC between 2010-2017 at two international high-volume pancreatectomy centers were compared. Cox proportional hazard modeling was utilized for multivariate analysis.

Results: We identified 193 DPS and 253 RAMPS during the study period. DPS was associated with higher rates of median estimated blood loss (500 vs. $300 \mathrm{cc}, \mathrm{P}<0.001)$, median total harvested lymph nodes (18 vs. 12, $\mathrm{P}<0.001)$ and $\mathrm{R} 0$ resection $(94.3 \%$ vs. $88.9 \%, \mathrm{P}=0.013)$. There were no differences in rates of postoperative pancreatic fistula $(16.5 \%$ vs. $17.8 \%, \mathrm{P}=1)$ or postoperative hemorrhage $(5.9 \%$ vs. $3.6 \%, \mathrm{P}=0.385)$ (DPS vs. RAMPS). After controlling for significant clinical pathological parameters, RAMPS was associated with non-superior recurrence-free survival (RFS) (HR 0.29; 95\% CI, 0.07-1.27, P=0.101) and overall-survival (HR 1.03; 95\% CI, 0.71-1.49, P=0.895) compared with DPS. Similar results were observed in node-positive patients.

Conclusions: RAMPS is safe and effective in the treatment of PDAC, but is not associated with an improvement in either RFS or overall-survival over DPS.

Keywords: Pancreatic cancer; distal pancreatosplenectomy

Submitted Apr 11, 2020. Accepted for publication May 22, 2020.

doi: $10.21037 / \mathrm{cco}-20-6$

View this article at: http://dx.doi.org/10.21037/cco-20-6 


\section{Introduction}

Left-sided pancreatic adenocarcinoma (PDAC) is a particularly deadly disease that is often considered more lethal than cancers arising from the head $(1,2)$. In some studies, authors have even questioned the role of surgery for left-sided PDAC $(3,4)$. However, long-term survival after surgical resection has been demonstrated (5-7), and surgery is accepted as an essential component in modern treatment protocols (8). Several variations of the standard distal pancreatosplenectomy (DPS) have been described, most notably the radical antegrade modular pancreatosplenectomy (RAMPS) by Strasberg in 2003, which attempts to achieve wider tangential margins and complete removal of the N1 lymph node basin in order to enhance oncologic results (9-12). Classically, RAMPS involves division of the pancreatic neck, dissection of the celiac lymph nodes, posterior dissection just anterior to the adrenal gland usually including Gerota's fascia, and splenectomy (9).

Since its initial description, RAMPS adoption has been uneven, though the procedure is preferred in many highvolume centers in Asia (13-16). PDAC's preponderance for origination in the head or uncinate (only $15 \%$ of PDAC arise in the body or tail) (2), heterogenous disease biology, and frequent early development of metastatic disease make robust comparison of DPS vs. RAMPS via randomized controlled trial impractical. Systemic reviews of published data have been performed but are also limited in size and scope $(17,18)$. Included studies are predominately small, single institution case series, none of which included more than 100 patients and less than half reported longterm survival data. While these pooled analyses have demonstrated improved R0-resection rate and greater lymph-node retrieval with RAMPS, it is unclear whether these oncologic surrogates translate into improved diseasefree and overall survival (OS), particularly in the era of increasingly effective loco-regional and systemic therapies $(19,20)$. This large, multi-institutional series of leftsided pancreatosplenectomy provides detailed, patientlevel clinical and survival data, and addresses many of the aforementioned shortcomings of previous reports. We present the following article in accordance with the STROBE reporting checklist (available at http://dx. doi. org/10. 21037/(cco-20-6).

\section{Methods}

\section{Patient selection and data acquisition}

All patients who underwent RAMPS and DPS for PDAC between 2010-2017 were identified from an international dual-institution database. This database included patients treated at the Johns Hopkins Hospital (Baltimore, MD, USA) and Changhai Hospital (Shanghai, China). Patients were excluded if they underwent a minimally invasive pancreatectomy or if they received neoadjuvant chemotherapy. The Institutional Review Boards at the participating institutions approved the study. Informed consent of study participants was not required by either institution due to the retrospective nature of the review. All RAMPS were performed at Changhai Hospital and all DPS were performed at Johns Hopkins Hospital throughout the study period as part of each institution's standard practice protocol. All RAMPS procedures included N1 lymphadenectomy (including celiac lymph nodes) and resection of Gerota's fascia for posterior RAMPS, components that were not routinely performed in DPS.

Standard demographic and clinicopathologic data were collected from the medical record including age, gender, race, type of pancreatectomy, estimated blood loss, and the presence of multivisceral resection. Specimen-specific characteristics including tumor size, grade, perineural invasion, and margin status were also noted. R1 margin status was defined as $<1 \mathrm{~mm}$ from the edge of the specimen. Complication data were abstracted from the medical record. OS was defined as the time interval between the date of surgery and the date of death. Recurrence-free survival (RFS) was similarly calculated as the interval between date of surgery and the date of first evidence of tumor recurrence. Surveillance protocols and indications for adjuvant chemotherapy type and duration were left to the discretion of the treating surgeon and medical oncologist.

\section{Statistical analysis}

All continuous variables were presented as mean with $98 \%$ confidence interval (CI) or median with interquartile ranges (IQRs) depending on data distribution. Categorical variables were presented as counts with percentile. The differences between continuous variables were evaluated with Welch's $t$-test or Wilcoxon test, and differences between categorical 
Table 1 Clinicopathological characteristics of patients who pancreatosplenectomy for pancreatic ductal adenocarcinoma

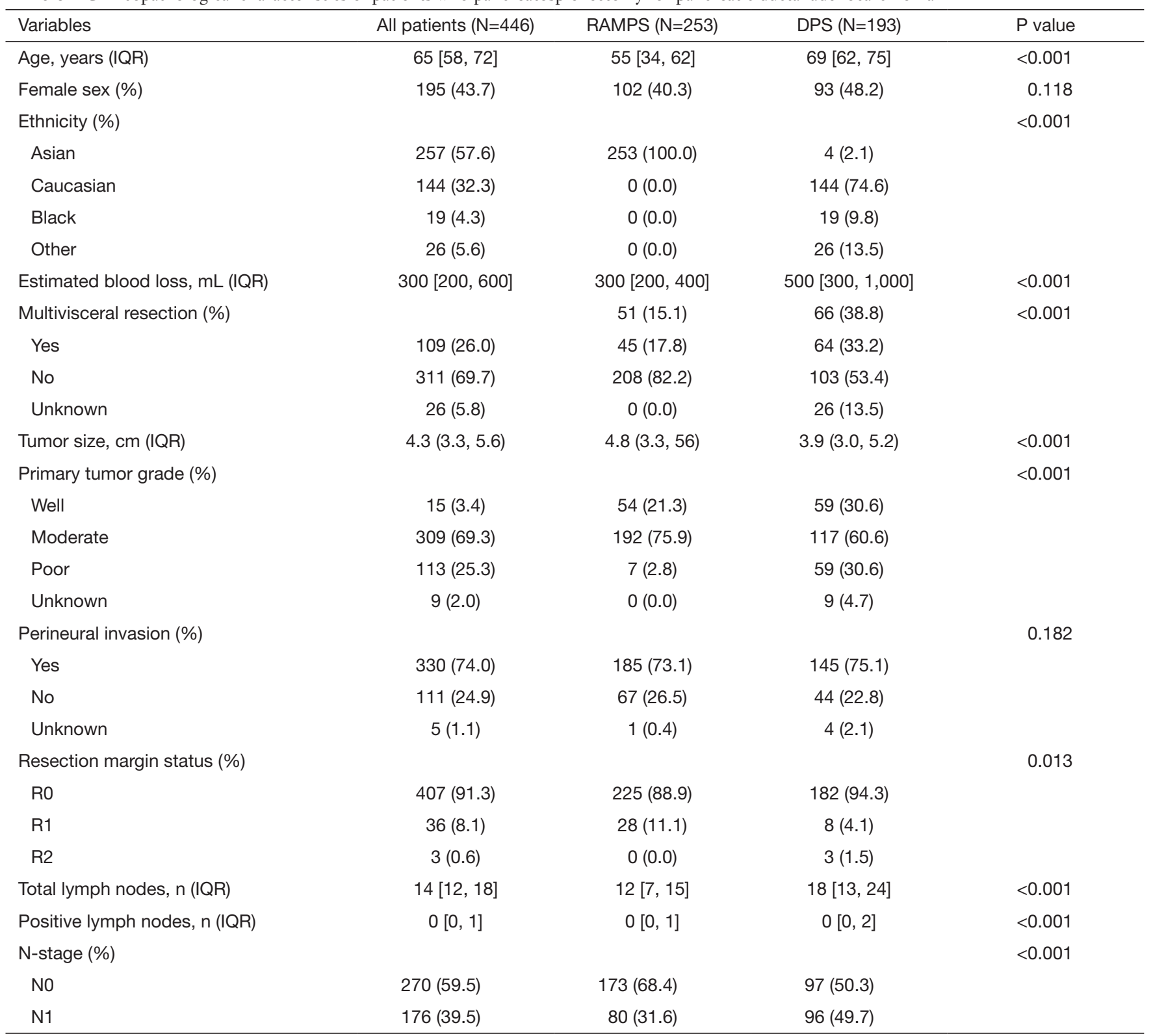

variables were evaluated with chi-square test or Fisher's exact test as appropriate. The primary outcomes, OS and RFS, were assessed with a multivariable Cox regression model. All patients were included for OS analysis. Patients who died without recurrence information were regarded as unevaluable and excluded for RFS analysis. Two-sided tests with $\mathrm{P}$ values less than 0.05 were considered statistically significant.

\section{Results}

A total of 446 patients underwent open pancreatosplenectomy without neoadjuvant chemotherapy during the study period (Table 1). Median patient age was 65 and the majority of patients were Asian $(\mathrm{n}=257,57.6 \%)$ and female $(\mathrm{n}=251$, $56.3 \%)$. Slightly over half (56.7\%) of the cohort underwent RAMPS. Median blood loss was higher in the DPS cohort 
Table 2 Postoperative complications and adjuvant therapy

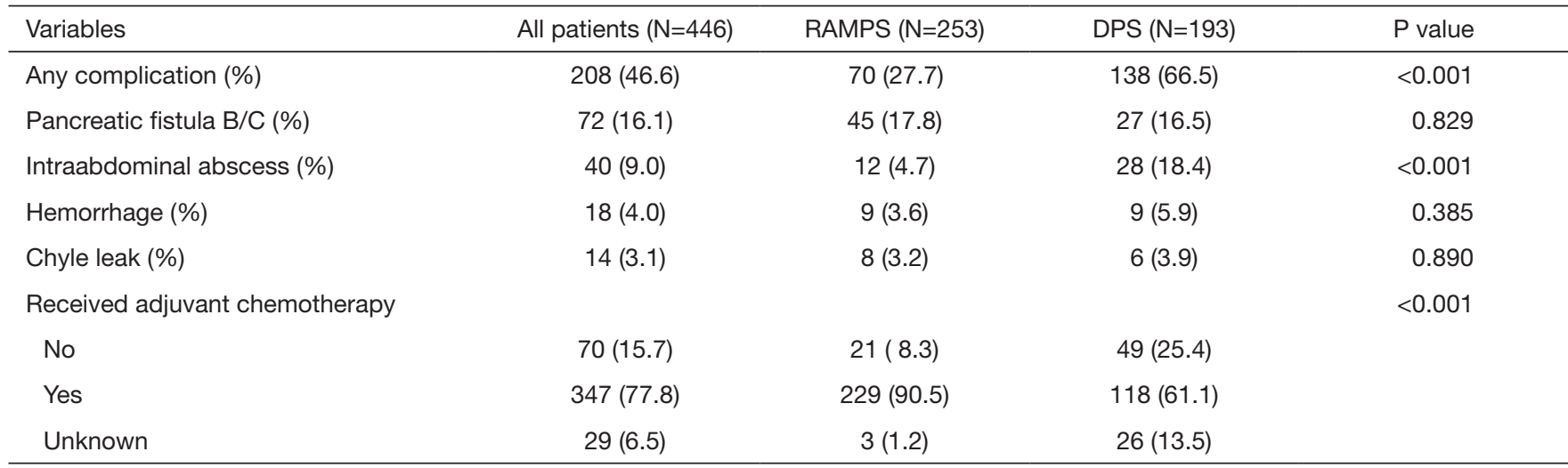
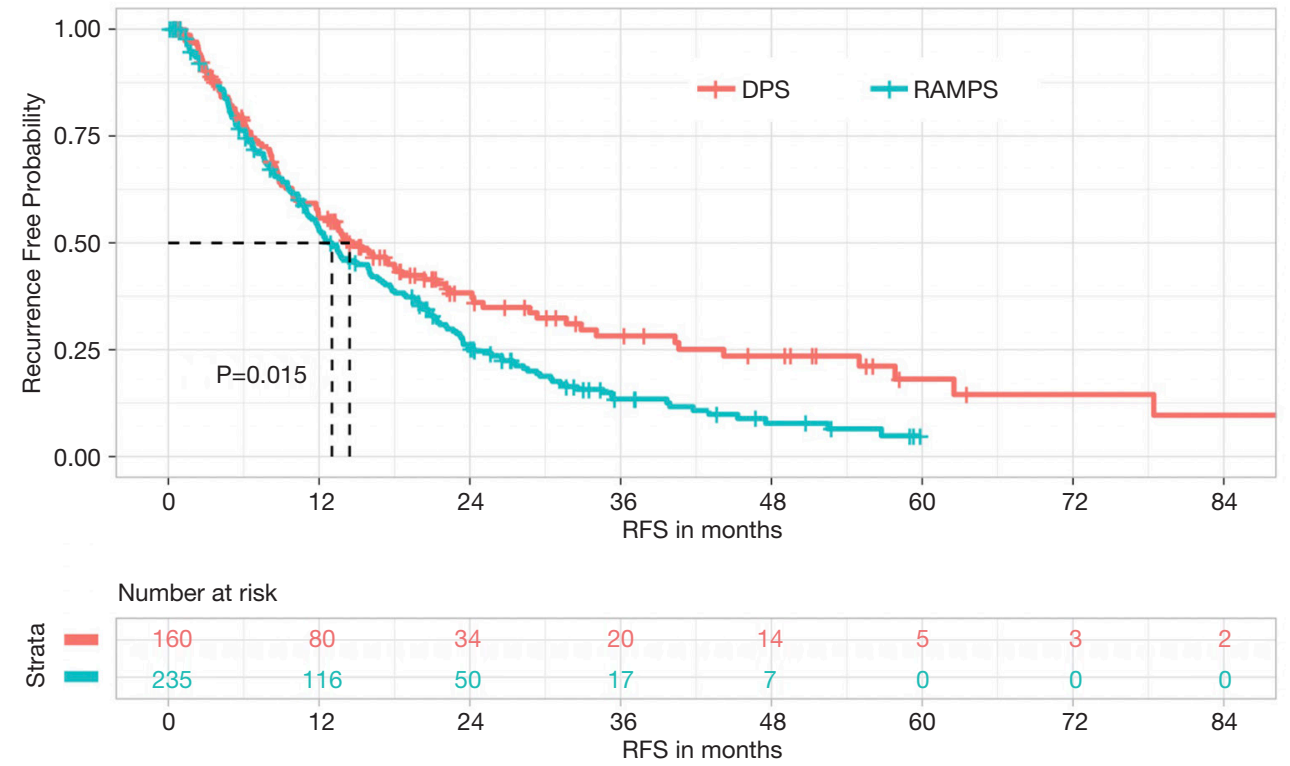

Figure 1 Kaplan-Meyer survival curves comparing recurrence-free survival in patients undergoing RAMPS and DPS for PDAC. RAMPS, radical antegrade modular pancreatosplenectomy; DPS, distal pancreatosplenectomy; PDAC, pancreatic adenocarcinoma.

(500 vs. $300 \mathrm{~mL}, \mathrm{P}<0.001$ ) as well as rates of multivisceral resection $(38.8 \%$ vs. $15.1 \%, \mathrm{P}<0.001)$. Tumor size as determined by pathological measurement was higher in the RAMPS cohort (48 vs. $39 \mathrm{~mm}, \mathrm{P}<0.001$ ). The margin status was R0 in a significant majority of patients in both cohorts, with a slightly higher rate in patients undergoing DPS ( $94.3 \%$ vs. $88.9 \%, \mathrm{P}=0.013)$. The median number of detected lymph nodes was also higher with DPS (18 vs. 12 nodes, $\mathrm{P}<0.001)$ and the majority of patients in both groups had node-negative disease.

Overall reported complication rates were higher in the DPS cohort $(66.5 \%$ vs. $27.7 \%, \mathrm{P}<0.001)$, however no difference in the rates of pancreatic fistula $(16.5 \%$ vs. $17.8 \%, \mathrm{P}=0.829)$, postoperative hemorrhage $(5.9 \%$ vs. $3.6 \%, \mathrm{P}=0.385)$, and chyle leak $(3.9 \%$ vs. $3.2 \%, \mathrm{P}=0.890)$ were noted (Table 2). More patients who underwent RAMPS received adjuvant chemotherapy as compared to the DPS group ( $90.5 \%$ vs. $61.1 \%, \mathrm{P}<0.001)$. Unfortunately, detailed data regarding the type and duration of adjuvant chemotherapy was not available.

\section{Factors affecting RFS}

Disease recurrence occurred in 291 (73.7\%) patients during 
Table 3 Hazard regression analysis of factors associated with recurrence-free survival

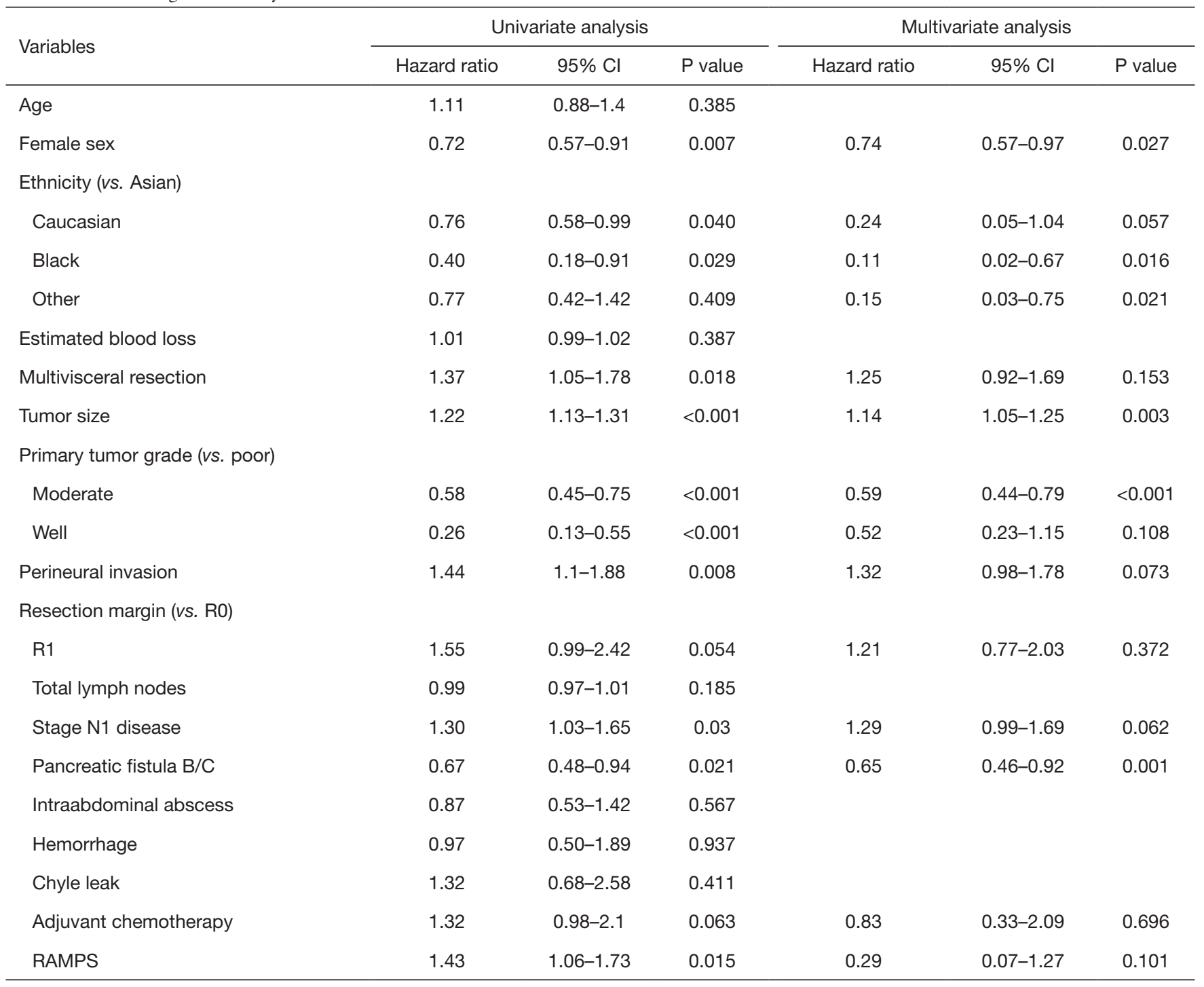

the study period (Figure 1). Several clinicopathologic factors were associated with improved RFS on multivariate analysis including female sex (HR 0.74; 95\% CI, 0.57-0.97, $\mathrm{P}=0.027$ ) and moderate tumor differentiation (HR 0.59; 95\% CI, 0.44-0.79, $\mathrm{P}<0.001$ ) (Table 3). Well-differentiated tumors also trended towards prolonged RFS but did not reach statistical significance. Poorer RFS was observed in patients with larger tumors (HR 1.14; 95\% CI, 1.05-1.25, $\mathrm{P}=0.003)$. Neither resection margin status nor the presence of nodal metastases was found to affect RFS. Additionally, patients who underwent RAMPS were not associated with an improvement in RFS in either univariate or multivariate analysis (HR 0.29; 95\% CI, 0.07-1.27, P=0.101).

\section{Factors affecting $O S$}

After a median follow-up of 24.3 months, 274 (61.4\%) patients in the entire cohort had died (Figure 2). The 1-, 3-, and 5 -year OS was $70.6 \%, 31.1 \%$, and $20.0 \%$, respectively. On univariate analysis, increased age, multivisceral resection, larger tumor size, poor tumor grade, perineural invasion, R1 resection margin, and $\mathrm{N} 1$ nodal status were associated with decreased OS (Table 4). On multivariate analysis, no patient factors adversely affected OS, while 

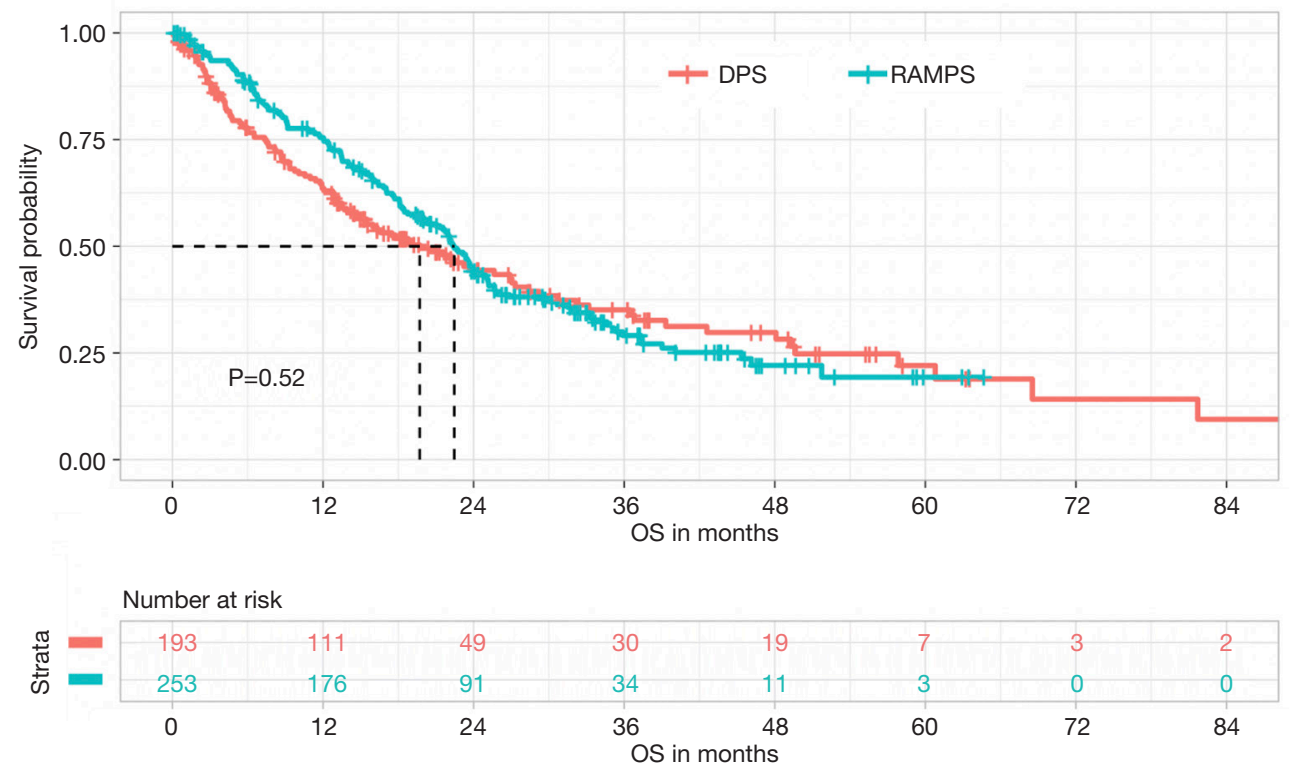

Figure 2 Kaplan-Meyer survival curves comparing overall survival in patients undergoing RAMPS and DPS for PDAC. RAMPS, radical antegrade modular pancreatosplenectomy; DPS, distal pancreatosplenectomy; PDAC, pancreatic adenocarcinoma.

certain pathologic factors maintained their association with a reduction in OS including increased tumor size (HR 1.61; 95\% CI, 1.06-1.28, $\mathrm{P}=0.002)$, $\mathrm{R} 1$ resection margin (HR 1.58; 95\% CI, 1.03-2.45, $\mathrm{P}=0.038$ ), and $\mathrm{N} 1$ disease (HR 1.39; 95\% CI, 1.03-1.89, P=0.031). When compared with poor-differentiation, moderate and well-differentiated tumor grades were also associated improved OS, though only moderate differentiation was statistically significant (HR 0.56; 95\% CI, 0.41-0.77, $\mathrm{P}<0.001$ ). After controlling for all other significant factors in the cox proportional hazard regression analysis, RAMPS was not associated with a difference in OS (HR 1.03; 95\% CI, 0.71-1.49, $\mathrm{P}=0.895$ ).

\section{Survival in node-positive patients}

After resection, 176 (39.5\%) patients were found to have lymph node metastases. Median OS was shorter in this cohort when compared with node-negative patients (16.7 vs. 23.9 months, $\mathrm{P}=0.002$ ) (Figure 3). However, even amongst patients with node-positive disease, performing RAMPS did not alter RFS or OS. During the study period, median RFS in N1 patients undergoing RAMPS was 10.4 months compared with 11.9 months in patients undergoing DPS $(\mathrm{P}=0.21)$. Median OS was also statistically equivalent between RAMPS and DPS in the node-positive cohort (16.3 vs. 16.4 months, $\mathrm{P}=0.71$ ).

\section{Discussion}

Surgery plays a vital role in the treatment of PDAC for appropriately selected patients. Despite high-quality data demonstrating a lack of oncologic benefit when performing concurrent extended lymphadenectomy with pancreaticoduodenectomy for right-sided PDAC $(21,22)$, many centers still consider RAMPS for left-sided lesions as potentially beneficial. This is the largest multi-institutional series of left-sided pancreatosplenectomy in the literature to date and includes more patients than several published systemic reviews and meta-analyses $(17,18)$. And while there is important heterogeneity between participating institutions, this case series design allows for detailed recurrence and survival data to be collected and compared across the study population.

Many RAMPS proponents indicate improved R0 resection rates and broader lymph node retrieval as evidence of the procedure's oncologic benefit. Indeed, the findings of higher R0 margin rates and more total lymph nodes harvested described in previous reports $(17,18)$ comparing the techniques were not reproduced in this study. This is most likely due to differences in the technical pathologic evaluation of surgical specimen between the two institutions. Pathologists evaluating DPS specimens utilized a standardized specimen handling protocol (23) which was not used for the RAMPS specimens, likely resulting in variable 
Table 4 Hazard regression analysis of factors associated with overall survival

\begin{tabular}{|c|c|c|c|c|c|c|}
\hline Variables & \multicolumn{3}{|c|}{ Univariate analysis } & \multicolumn{3}{|c|}{ Multivariate analysis } \\
\hline Age & 1.30 & $1.02-1.64$ & 0.032 & 1.07 & $0.8-1.44$ & 0.637 \\
\hline Female sex & 0.85 & $0.67-1.08$ & 0.184 & & & \\
\hline \multicolumn{7}{|l|}{ Ethnicity (vs. Asian) } \\
\hline Black & 0.78 & $0.38-1.58$ & 0.482 & & & \\
\hline Other & 1.37 & $0.81-2.33$ & 0.241 & & & \\
\hline Estimated blood loss (per 100 mL) & 1.01 & $1-1.03$ & 0.052 & 1.01 & $1-1.03$ & 0.165 \\
\hline Multivisceral resection & 1.53 & $1.17-1.99$ & 0.002 & 1.26 & $0.91-1.74$ & 0.162 \\
\hline Moderate & 0.58 & $0.45-0.75$ & $<0.001$ & 0.56 & $0.41-0.77$ & $<0.001$ \\
\hline Well & 0.27 & $0.12-0.63$ & 0.002 & 0.54 & $0.21-1.37$ & 0.194 \\
\hline Perineural invasion & 1.64 & $1.23-2.2$ & 0.001 & 1.24 & $0.89-1.74$ & 0.209 \\
\hline \multicolumn{7}{|l|}{ Resection margin } \\
\hline $\mathrm{R} 1$ & 2.06 & $1.4-3.01$ & $<0.001$ & 1.58 & $1.03-2.45$ & 0.038 \\
\hline Total lymph nodes & 0.99 & $0.97-1.01$ & 0.23 & & & \\
\hline Stage N1 disease & 1.48 & $1.16-1.88$ & 0.001 & 1.39 & $1.03-1.89$ & 0.031 \\
\hline Pancreatic fistula B/C & 0.82 & $0.58-1.15$ & 0.242 & & & \\
\hline
\end{tabular}

margin status and a lower reported total lymph node harvest in these patients. This is due to regional and institutional pathology practice variation and does not reflect the true scope of lymphadenectomy for the respective operations. While the actual number of lymph nodes removed during RAMPS is likely underreported, it is important to note that the median 12 (IQR, 7-15) harvested lymph nodes observed in the RAMPS cohort meets the minimum threshold set by the International Study Group for Pancreatic Surgery (24) and is greater than the median lymph node retrieval (9 lymph nodes) reported in Strasberg's original description of RAMPS. Additionally, margin status is an imperfect surrogate for oncologic efficacy, and the ultimate independent effect of the microscopic resection margin on OS and RFS in PDAC is debated, and in some reports have shown not to significantly impact survival outcomes (25). This is likely due to the introduction of increasingly effective systemic treatment modalities which can improve survival even in the setting of an R1 resection (20).

The overall complication rate of $46.6 \%$ is comparable to average rates of $40 \%$ reported in other series (18). The increased incidence of overall complications observed in DPS patients was likely due to more robust reporting of Grade 1 complications (e.g., uncomplicated UTI). This is secondary to variability in how data for minor complications are reported at each institution, likely underreporting 

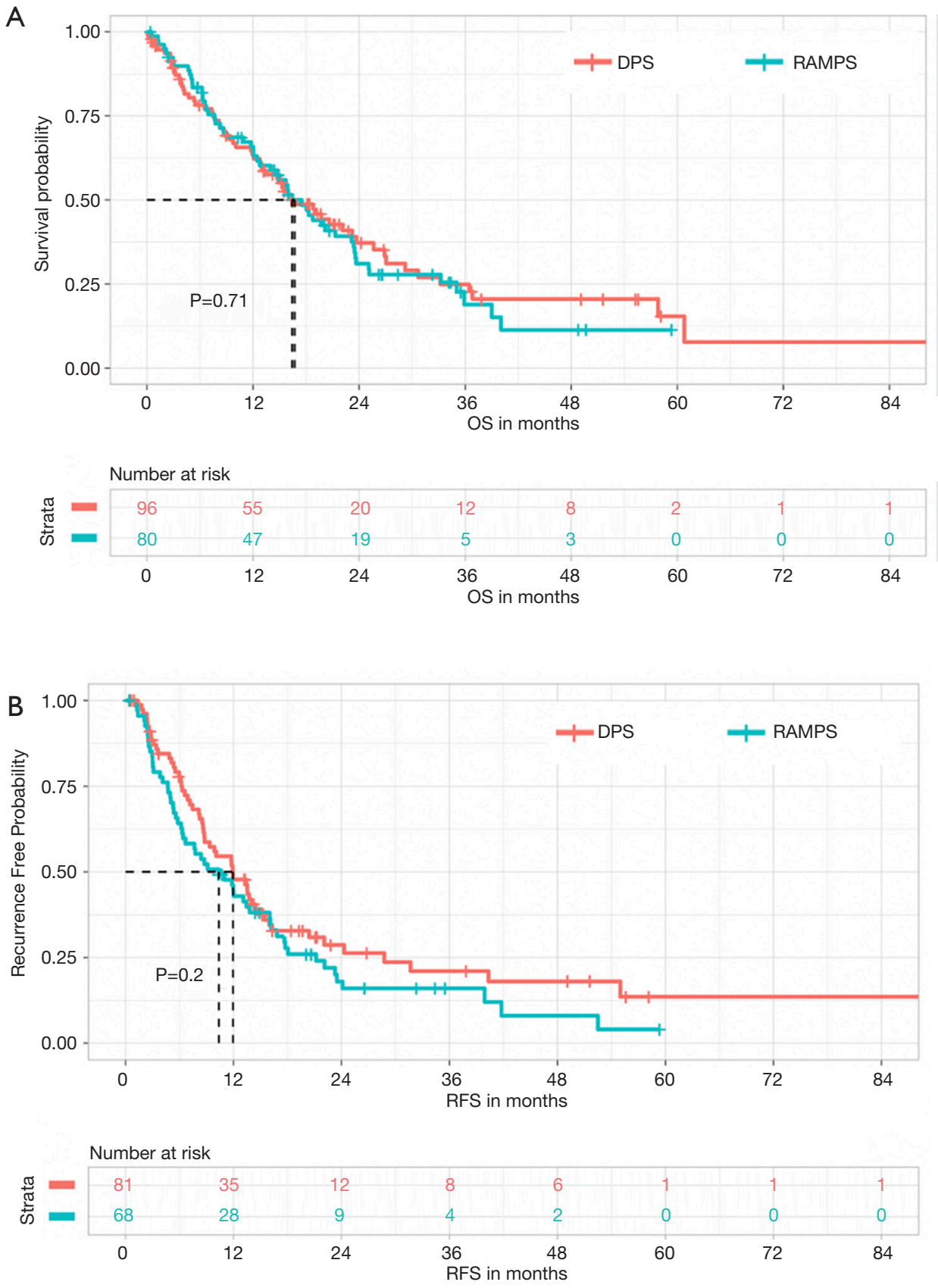

Figure 3 Kaplan-Meyer survival curves comparing (A) overall survival and (B) recurrence-free survival in node-positive patients undergoing RAMPS and DPS for PDAC. RAMPS, radical antegrade modular pancreatosplenectomy; DPS, distal pancreatosplenectomy; PDAC, pancreatic adenocarcinoma.

these complications for patients undergoing RAMPS. Unfortunately, the transfusion rate in the each respective cohort was not available for inclusion in the multivariable analysis. The only significant difference in major complication between the procedures was observed in rates of postoperative intraabdominal abscess. This increased rate of deep-organ space infections may be related to more frequent multivisceral resections in the DPS group, which is also likely the result of local surgical practice patterns. Alternatively, unmeasured patient-related microbiome, 
genetic, environmental, or other health-system factors may be driving this difference as the RAMPS and DPS cohorts were treated in disparate physical locations by different healthcare teams. Similar health system-related factors likely propelled the differences in adjuvant chemotherapy rates between the groups.

Without knowing specific drug, dosing, and duration data it is impossible to extrapolate the relative effects of this chemotherapy between the two surgical groups. The rate of adjuvant chemotherapy administration observed in the DPS group is similar to the nearly $70 \%$ administration rate reported after all pancreatectomies for adenocarcinoma in the United States (26). The increased adjuvant therapy rate observed in the RAMPS cohort is likely due to frequent use of single-agent treatment regimens (such as S1) which are generally better tolerated, as well as standardized adjuvant therapy in China. If RAMPS were more oncologically effective, it would be expected that the high rates of adjuvant therapy $(90.5 \%)$ observed in this cohort would bias the survival data in favor of improved OS. This was not observed in the final survival analysis.

The primary study result of no difference in survival between the RAMPS and DPS cohort supports the findings of several other smaller studies $(13,27,28)$ as well as systemic reviews $(17,18)$. While this has been demonstrated repeatedly in broader study populations, some authors have suggested that extended resection and regional lymphadenectomy may be selectively beneficial in node positive patients (22). Our subgroup analysis of N1 patients did not demonstrate a survival benefit with RAMPS in this population. Survival in this group is more likely determined by overall disease biology and the presence of undetected micrometastatic disease, rather than the magnitude of local resection. This concept is similar to that found in other malignancies such as breast cancer, where more extensive local and regional resections do not necessarily result in improved long-term survival (29,30).

In addition to those mentioned above, the current study has several limitations. As with all retrospective studies, uncontrolled treatment allocation bias is potentially present, however mitigated by the fact that each institution only performed one type of resection. Other significant patient (genetic, socioeconomic, etc.) and health system-related (postoperative treatment, adjuvant therapy protocols, etc.) factors that may affect survival also likely differed between the groups, however were controlled for when possible. As mentioned previously, non-standardized postoperative specimen evaluation makes comparison of pathologic factors such as margin status and extent of nodal harvest imprecise. Additionally, the type and duration of adjuvant chemotherapy was not collected and therefore their particular effects could not be assessed.

\section{Conclusions}

RAMPS has an acceptable safety and efficacy profile, however it is not associated with an improvement in either RFS or OS over DPS in the treatment of PDAC. The technical approach to pancreatosplenectomy should be selected based on surgeon experience and comfort, with the understanding that long-term oncologic outcomes are primarily influenced by disease biology and systemic therapy.

\section{Acknowledgments}

Funding: This study was supported by Project of Clinical Medical Centers (Pancreatic Disease) in Shanghai $2017 Z Z 01009$.

\section{Footnote}

Reporting Checklist: The authors have completed the STROBE reporting checklist. Available at http://dx.doi. org/10.21037/cco-20-6

Data Sharing Statement: Available at http://dx.doi. org/10.21037/сco-20-6

Peer Review File: Available at http://dx. doi. org/10.21037/ cco-20-6

Conflicts of Interest: All authors have completed the ICMJE uniform disclosure form (available at http://dx. doi. org/10. 21037/cco-20-6). JH serves as an unpaid editorial board member of Chinese Clinical Oncology. The other authors have no other conflicts of interest to declare.

Ethical Statement: The authors are accountable for all aspects of the work in ensuring that questions related to the accuracy or integrity of any part of the work are appropriately investigated and resolved. The study was conducted in accordance with the Declaration of Helsinki (as revised in 2013). The Institutional Review Boards at 
the participating institutions approved the study. Informed consent of study participants was not required by either institution due to the retrospective nature of the review.

Open Access Statement: This is an Open Access article distributed in accordance with the Creative Commons Attribution-NonCommercial-NoDerivs 4.0 International License (CC BY-NC-ND 4.0), which permits the noncommercial replication and distribution of the article with the strict proviso that no changes or edits are made and the original work is properly cited (including links to both the formal publication through the relevant DOI and the license). See: https://creativecommons.org/licenses/by-nc-nd/4.0/.

\section{References}

1. Niederhuber JE, Brennan MF, Menck HR. The National Cancer Data Base report on pancreatic cancer. Cancer 1995;76:1671-7.

2. Artinyan A, Soriano PA, Prendergast $\mathrm{C}$, et al. The anatomic location of pancreatic cancer is a prognostic factor for survival. HPB (Oxford) 2008;10:371-6.

3. Baumel H, Huguier M, Manderscheid JC, et al. Results of resection for cancer of the exocrine pancreas: a study from the French Association of Surgery. Br J Surg 1994;81:102-7.

4. Fabre JM, Houry S, Manderscheid JC, et al. Surgery for left-sided pancreatic cancer. Br J Surg 1996;83:1065-70.

5. Dalton RR, Sarr MG, van Heerden JA, et al. Carcinoma of the body and tail of the pancreas: is curative resection justified? Surgery 1992;111:489-94.

6. Yamamoto J, Saiura A, Koga R, et al. Improved survival of left-sided pancreas cancer after surgery. Jpn J Clin Oncol 2010;40:530-6.

7. van Gulik TM, Nakao A, Obertop H. Extended resection for pancreatic adenocarcinoma. HPB (Oxford) 2002;4:101-3.

8. NCCN Practica Guidelines In Clinical Oncology: Pancreatic Adenocarcinoma v 1.2019. 2018. Available online: https://www.nccn.org/professionals/physician_gls/ pdf/pancreatic.pdf. Accessed December 252018.

9. Strasberg SM, Drebin JA, Linehan D. Radical antegrade modular pancreatosplenectomy. Surgery 2003;133:521-7.

10. Strasberg SM, Linehan DC, Hawkins WG. Radical antegrade modular pancreatosplenectomy procedure for adenocarcinoma of the body and tail of the pancreas: ability to obtain negative tangential margins. J Am Coll Surg 2007;204:244-9.
11. Strasberg SM, Fields R. Left-sided pancreatic cancer: distal pancreatectomy and its variants: radical antegrade modular pancreatosplenectomy and distal pancreatectomy with celiac axis resection. Cancer J 2012;18:562-70.

12. Mitchem JB, Hamilton N, Gao F, et al. Long-term results of resection of adenocarcinoma of the body and tail of the pancreas using radical antegrade modular pancreatosplenectomy procedure. J Am Coll Surg 2012;214:46-52.

13. Abe T, Ohuchida K, Miyasaka Y, et al. Comparison of Surgical Outcomes Between Radical Antegrade Modular Pancreatosplenectomy (RAMPS) and Standard Retrograde Pancreatosplenectomy (SPRS) for Left-Sided Pancreatic Cancer. World J Surg 2016;40:2267-75.

14. Murakawa M, Aoyama T, Asari M, et al. The shortand long-term outcomes of radical antegrade modular pancreatosplenectomy for adenocarcinoma of the body and tail of the pancreas. BMC Surg 2015;15:120.

15. Han DH, Kang CM, Lee WJ, et al. A five-year survivor without recurrence following robotic anterior radical antegrade modular pancreatosplenectomy for a wellselected left-sided pancreatic cancer. Yonsei Med J 2014;55:276-9.

16. Park HJ, You DD, Choi DW, et al. Role of radical antegrade modular pancreatosplenectomy for adenocarcinoma of the body and tail of the pancreas. World J Surg 2014;38:186-93.

17. Cao F, Li J, Li A, et al. Radical antegrade modular pancreatosplenectomy versus standard procedure in the treatment of left-sided pancreatic cancer: A systemic review and meta-analysis. BMC Surg 2017;17:67.

18. Zhou Y, Shi B, Wu L, et al. A systematic review of radical antegrade modular pancreatosplenectomy for adenocarcinoma of the body and tail of the pancreas. HPB (Oxford) 2017;19:10-5.

19. Hazard L. The role of radiation therapy in pancreas cancer. Gastrointest Cancer Res 2009;3:20-8.

20. Conroy T, Hammel P, Hebbar M, et al. FOLFIRINOX or Gemcitabine as Adjuvant Therapy for Pancreatic Cancer. N Engl J Med 2018;379:2395-406.

21. Farnell MB, Pearson RK, Sarr MG, et al. A prospective randomized trial comparing standard pancreatoduodenectomy with pancreatoduodenectomy with extended lymphadenectomy in resectable pancreatic head adenocarcinoma. Surgery 2005;138:618-28; discussion 628-30.

22. Bassi C, Salvia R, Butturini G, et al. Value of regional lymphadenectomy in pancreatic cancer. HPB (Oxford) 
2005;7:87-92.

23. Westra WH, Hruban RH, Phelps TH, et al. Surgical pathology dissection: an illustrated guide. 2nd Edition. Berlin: Springer, 2002.

24. Tol JA, Gouma DJ, Bassi C, et al. Definition of a standard lymphadenectomy in surgery for pancreatic ductal adenocarcinoma: a consensus statement by the International Study Group on Pancreatic Surgery (ISGPS). Surgery 2014;156:591-600.

25. Yamamoto T, Uchida Y, Terajima H. Clinical impact of margin status on survival and recurrence pattern after curative-intent surgery for pancreatic cancer. Asian J Surg 2019;42:93-9.

26. Ma SJ, Oladeru OT, Miccio JA, et al. Association of Timing of Adjuvant Therapy With Survival in Patients With Resected Stage I to II Pancreatic Cancer. JAMA Netw Open 2019;2:e199126.

Cite this article as: Sham JG, Guo S, Ding D, Shao Z, Wright M, Jing W, Yin LD, Zhang Y, Gage MM, Zhou Y, Javed A, Burkhart RA, Zhou X, Weiss MJ, He T, Li G, Cameron JL, Hu X, Wolfgang CL, Jin G, He J. Radical antegrade modular pancreatosplenectomy versus standard distal pancreatosplenectomy for pancreatic cancer, a dual-institutional analysis. Chin Clin Oncol 2020;9(4):54. doi: 10.21037/cco-20-6
27. Latorre M, Ziparo V, Nigri G, et al. Standard retrograde pancreatosplenectomy versus radical antegrade modular pancreatosplenectomy for body and tail pancreatic adenocarcinoma. Am Surg 2013;79:1154-8.

28. Kim EY, You YK, Kim DG, et al. Initial experience with radical antegrade modular pancreatosplenectomy in a single institution. Ann Surg Treat Res 2016;91:29-36.

29. Giuliano A, Hunt K, Ballman K, et al. Axillary dissection vs no axillary dissection in women with invasive breast cancer and sentinel node metastasis: a randomized clinical trial. JAMA 2011;305:569-75.

30. Fisher B, Anderson S, Bryant J, et al. Twenty-year followup of a randomized trial comparing total mastectomy, lumpectomy, and lumpectomy plus irradiation for the treatment of invasive breast cancer. $\mathrm{N}$ Engl J Med 2002;347:1233-41. 